Objectives Aim of the present study was to evaluate the seroprevalence of HSV-1, and HSV-2 infections among various population groups in Siberia, Russia.

Methods A sample of 1014 persons aged 14-64 years (47\% males) were randomly selected from the general population in urban Novosibirsk (predominantly Caucasians) and rural regions of Tyva and Altai Republic (predominantly persons of Asian origin). The study includes data obtained in 1994-2005. The current investigation was performed as an optional fragment of international WHO MONICA study, and HAPIEE study (Wellcome Trust Fund 064947/Z/01/Z and 081081/Z/06/Z). Sera were tested for IgG antibodies to HSV infections with type-specific ELISA (VectoHSV-1 IgG, VectoHSV-2 IgG, Vector-Best, Novosibirsk, Russia).

Results The overall prevalence of HSV-2 positivity was $21 \%$ being higher in females $(26.4 \%)$ compared to males $(17.0 \%, p=0.01)$. Seroprevalence rates increased with age from $0 \%$ at $14-17$ years to $22.8 \%$ at $55-64$ years in males and from $1.8 \%$ at $14-17$ years to $31.6 \%$ at 55-64 years in females. Neither difference was observed between urban and rural population, nor in surveyed persons of Caucasian and Asian origin. Ten-year trend showed a decrease in the frequency of HSV-2 detection equally in different populations by $20 \%$. HSV-1 seroprevalence was uniformly high in each population group (99-100\%). In conclusion, HSV-2 is common in Russia with the prevalence rates close to USA and Scandinavian countries. The sex and age distribution of the infection in Russia is similar to other populations. In Siberia, ethnicity and standard of living do not influence HSV-2 prevalence. However, HSV-1 infection rates may influence HSV-2 infection distribution by cross-reactive immunity patterns.

\section{P1-S1.21 INCIDENCE RATE OF HERPES SIMPLEX VIRUS TYPE 2 (HSV-2) IN THE USA, 1988-2008}

doi:10.1136/sextrans-2011-050108.21

S Gerver. Imperial College London, London, UK

Background HSV-2 infection remains a major public health problem in the USA, where the prevalence is $16.2 \%$ in persons aged $14-49$. In this report, we provide national estimates of new HSV-2 infections in the USA and examine trends in incidence over the past 2 decades. Methods A simple catalytic model was used to estimate HSV-2 force of infection (FOI), defined as the incidence of HSV-2 in the susceptible population, from National Health and Nutrition Examination Surveys (NHANES) in the USA from 1988 to 2008. HSV-2 serology was included in the nationally representative surveys during 1988-1994 (NHANES III) and in continuous NHANES from 1999 to 2008. Incidence rates and trends over time were estimated by age, sex and race/ethnic group for non-Hispanic whites, non-Hispanic blacks, and Mexican-Americans.

Results HSV-2 FOI rates were highest in non-Hispanic blacks and lowest in non-Hispanic whites, with the FOI among 25-year-old non-Hispanic black women more than 13 times greater than white men of the same age. Rates were highest between ages 25 and 35 years for all sex-race/ethnicity groups (peak in non-Hispanic white and black women aged 25 years, in non-Hispanic white and black men aged 35 years and in Mexican-American women in their early-30s) except for Mexican-American men, for whom there was less variation in FOI by age. Over the last 20 years, age-adjusted FOI rates were stable for the sex-race/ethnicity groups examined except for non-Hispanic white and Mexican-American women, in which there were decreases after 2002 and 2001, respectively. FOI rates were lower in men than women among non-Hispanic blacks and Mexican-Americans, and in non-Hispanic whites prior to 2002. Overall, the estimated number of new HSV-2 infections in 2007-2008, among these three groups, aged 14-49 years, was in excess of 750000 , with $48 \%(\sim 360000)$ occurring in women and
$52 \%(\sim 392000)$ in men. Nearly half (45\%) of all infections occurred in persons under 25 years old ( $34 \%$ and $57 \%$ of infections in men and women, respectively).

Conclusion Risk of HSV-2 infection varies substantially across the US adult population, with the highest risk among non-Hispanic black men and women. Information on the detailed distribution of new infections can help improve the efficiency of interventions. In addition, although we found evidence for substantial reductions in the risk of HSV-2 among some race/ethnicity groups, FOI in groups with the highest rates has not changed in 20 years.

\section{P1-S1.22 HSV-2 PREVALENCE ACROSS A PASTORAL LANDSCAPE: TRANSMISSION AND TRANSITION AMONG THE HIMBA AND TJIMBA POPULATIONS}

doi:10.1136/sextrans-2011-050108.22

A Hazel, B Foxman, B Low. University of Michigan, Ann Arbor, USA

Background Sexual partnerships take multiple forms among the Himba and Tjimba people living in Northwestern Namibia, including polygynous and monogamous marriage, and long- and short-term extra-marital partnerships. We hypothesised that HSV-2 prevalence would be higher in regions that are connected to local urban areas, because these should be sites of more frequent anonymous partner exchange.

Methods In 2009, we surveyed 30 villages, obtaining HSV-2 status (using the BioKit Rapid Test for HSV-2) and demographics, home village, recent travel patterns and sex partnerships $(n=446)$. We estimated prevalence (a) for the entire population (b) for all women and men and c) for individual villages. Logistic regression models were fit to test the correlation between risk of infection and travel patterns.

Results Overall HSV-2 prevalence was $36.5 \%$, with higher rates among women (50.5\%) than men (23.3\%). In contrast to expectation, the highest HSV-2 prevalences were found in some of the most remote regions. To explore this further, we built a network model that superimposes travel patterns onto sex partnership data. Using this model, we observed that older travel corridors (eg, travel from home village to extended-family associated villages) were more associated with higher HSV-2 transmission than newer corridors (eg, travel to local cities to hang out). Ethnographic interviews suggest that differences in the nature of relationships that occur in urban areas (eg, lower contact frequency, higher condom use) may explain the difference between observed and predicted.

Conclusions In this population cultural and economic transitions that lead to more travel into urban areas were not associated with increase in HSV2 prevalence, probably because these relationships are deemed more risky. Shifting attitudes about sex and sex partnerships are likely to affect future STD transmission in this population.

\section{Epidemiology poster session 1: STI trends: syndromic approach \\ P1-S1.23 TREND OF SEXUALLY TRANSMITTED DISEASES DIAGNOSED BY SYNDROMIC APPROACH IN AN INSTITUTE SETTING}

doi:10.1136/sextrans-2011-050108.23

A Kanwar. Postgraduate Institute of Medical Education and Research, Chandigarh, India

Background The paradigm of research in sexually transmitted diseases (STD) has recently shifted to HIV/AIDS. Recent trend of sexually transmitted diseases in India is lacking. 